\title{
Solvability of a Class of Higher-Order Fractional Two-Point Boundary Value Problem
}

\author{
Xiangshan Kong ${ }^{1}$ and Haitao $\mathrm{Li}^{2}$ \\ ${ }^{1}$ Basic Science Department, Qingdao Binhai University, Qingdao 266555, China \\ ${ }^{2}$ School of Control Science and Engineering, Shandong University, Jinan 250061, China \\ Correspondence should be addressed to Haitao Li; haitaoli09@gmail.com
}

Received 3 September 2013; Accepted 17 October 2013; Published 2 January 2014

Academic Editors: A. Masiello and L. Xu

Copyright (c) $2014 \mathrm{X}$. Kong and H. Li. This is an open access article distributed under the Creative Commons Attribution License, which permits unrestricted use, distribution, and reproduction in any medium, provided the original work is properly cited.

\begin{abstract}
This paper investigates the solvability of a class of higher-order fractional two-point boundary value problem (BVP), and presents several new results. First, Green's function of the considered BVP is obtained by using the property of Caputo derivative. Second, based on Schaefer's fixed point theorem, the solvability of the considered BVP is studied, and a sufficient condition is presented for the existence of at least one solution. Finally, an illustrative example is given to support the obtained new results.
\end{abstract}

\section{Introduction}

Fractional differential equations (FDEs) have been of great interest recently. This is due to the development of the theory of fractional calculus itself as well as its applications [1-3]. Apart from diverse areas of mathematics, fractional differential equations arise in engineering, technology, biology, chemical process, and many other branches of science. The first issue for the theory of fractional differential equations is the existence of solutions to kinds of boundary value problems (BVPs), which has been studied recently by many scholars [4-18], and lots of excellent results have been obtained by means of fixed-point theorems, Leray-Schauder theory, upper and lower solutions technique, and so forth.

It should be pointed out that as an important branch of fractional differential equations, higher-order FDEs have been studied in a series of recent works [19-23]. In [19], higher-order fractional heat-type equations were investigated and some interesting properties on the solution to this type of equations were presented. Goodrich [21] considered another kind of higher-order fractional differential equations and presented some results on the existence of one positive solution.

In this paper, we study the following higher-order fractional two-point boundary value problem:

$$
{ }^{c} D_{0+}^{\alpha} x(t)=f(t, x(t)), \quad t \in[0,1],
$$

$$
x(0)=x(1)=x^{\prime \prime}(0)=x^{\prime \prime}(1)=0,
$$

where $3<\alpha<4, f:[0,1] \times R \rightarrow R$, and ${ }^{c} D_{0+}^{\alpha}$ is the Caputo derivative. To our best knowledge, there are fewer results on the existence of solutions to BVP (1). Firstly, we establish Green's function for BVP (1) by using the property of Caputo derivative. Secondly, based on Schaefer's fixed point theorem, we present a sufficient condition for the existence of at least one solution. Throughout this paper, we assume that the nonlinearity $f:[0,1] \times R \rightarrow R$ is continuous. Moreover, let $E=C[0,1]$ with the norm $\|x\|_{\infty}=\max _{t \in[0,1]}|x(t)|$. Then, $E$ is a Banach space.

The rest of this paper is structured as follows. Section 2 contains some preliminaries on the Caputo derivative. Section 3 investigates the existence of solutions to BVP (1) and presents the main results of this paper.

\section{Preliminaries}

In this section, we give some necessary preliminaries on the Caputo derivative, which will be used in the sequel. For details, please refer to [1-3] and the references therein. 
Definition 1 (see [3]). The Riemann-Liouville fractional integral of order $\alpha>0$ of a function $y:(0,+\infty) \rightarrow \mathbb{R}$ is given by

$$
I_{0+}^{\alpha} y(t)=\frac{1}{\Gamma(\alpha)} \int_{0}^{t}(t-s)^{\alpha-1} y(s) d s,
$$

provided that the right side is pointwise defined on $(0,+\infty)$.

Definition 2 (see [3]). The Caputo fractional derivative of order $\alpha>0$ of a continuous function $y:(0,+\infty) \rightarrow \mathbb{R}$ is given by

$$
{ }^{c} D_{0+}^{\alpha} y(t)=\frac{1}{\Gamma(n-\alpha)} \int_{0}^{t} \frac{y^{(n)}(s)}{(t-s)^{\alpha-n+1}} d s,
$$

where $n=[\alpha]+1$, provided that the right side is pointwise defined on $(0,+\infty)$.

One can easily obtain the following property from the definition of Caputo derivative.

Proposition 3 (see [3]). Let $\alpha>0$. Assume that $y,{ }^{c} D_{0+}^{\alpha} y \in$ $L(0,1)$. Then the following equality holds:

$$
I_{0+}^{\alpha c} D_{0+}^{\alpha} y(t)=y(t)+C_{0}+C_{1} t+\cdots+C_{n-1} t^{n-1}
$$

for some $C_{i} \in R, i=0,1, \ldots, n-1$, where $n=[\alpha]+1$.

Finally, we give Schaefer's fixed point theorem.

Lemma 4 (see [24]). Let $E$ be a Banach space, and $H: E \rightarrow$ $E$ is a completely continuous operator. If the set $S:=\{x \in E \mid$ $x=\lambda H x, \lambda \in(0,1)\}$, is bounded, then $H$ has at least a fixed point.

\section{Main Results}

In this section, we first convert BVP (1) into an equivalent operator equation and then present some new results on the existence of solutions to BVP (1).

Firstly, we convert BVP (1) into an equivalent operator equation.

Lemma 5. Given $h \in C[0,1]$, the unique solution of

$$
\begin{gathered}
{ }^{c} D_{0+}^{\alpha} x(t)=h(t), \quad t \in[0,1], \\
x(0)=x(1)=x^{\prime \prime}(0)=x^{\prime \prime}(1)=0
\end{gathered}
$$

is

$$
x(t)=\int_{0}^{1} G(t, s) h(s) d s
$$

where

$$
G(t, s)=\frac{1}{\Gamma(\alpha)}\left\{\begin{array}{c}
(t-s)^{\alpha-1}-t(1-s)^{\alpha-1} \\
+\frac{1}{6}(\alpha-2)(\alpha-1) \\
\times\left(t-t^{3}\right)(1-s)^{\alpha-3}, \\
0 \leq s \leq t \leq 1, \\
-t(1-s)^{\alpha-1}+\frac{1}{6}(\alpha-2) \\
\times(\alpha-1)\left(t-t^{3}\right)(1-s)^{\alpha-3}, \\
0 \leq t<s \leq 1 .
\end{array}\right.
$$

Proof. Assume that $x(t)$ satisfies (5). Then, from Proposition 3, we have

$$
x(t)=I_{0+}^{\alpha} h(t)+c_{0}+c_{1} t+c_{2} t^{2}+c_{3} t^{3}, \quad c_{0}, c_{1}, c_{2}, c_{3} \in R .
$$

From the boundary value condition $x(0)=x(1)=0$, one can see that

$$
\begin{aligned}
c_{0}=0, \quad c_{1}+c_{2}+c_{3} & =-\left.I_{0+}^{\alpha} h(t)\right|_{t=1} \\
& =-\frac{1}{\Gamma(\alpha)} \int_{0}^{1}(1-s)^{\alpha-1} h(s) d s
\end{aligned}
$$

Thus, we have

$$
x(t)=\frac{1}{\Gamma(\alpha)} \int_{0}^{t}(t-s)^{\alpha-1} h(s) d s+c_{1} t+c_{2} t^{2}+c_{3} t^{3}
$$

which together with the boundary value condition $x^{\prime \prime}(0)=$ $x^{\prime \prime}(1)=0$ yields that

$$
\begin{aligned}
x^{\prime}(t)= & \frac{1}{\Gamma(\alpha-1)} \\
& \times \int_{0}^{t}(t-s)^{\alpha-2} h(s) d s+c_{1}+2 c_{2} t+3 c_{3} t^{2},
\end{aligned}
$$

$$
\begin{aligned}
& x^{\prime \prime}(t)=\frac{1}{\Gamma(\alpha-2)} \int_{0}^{t}(t-s)^{\alpha-3} h(s) d s+2 c_{2}+6 c_{3} t \\
& c_{2}=0, \quad c_{3}=-\frac{1}{6} \frac{1}{\Gamma(\alpha-2)} \int_{0}^{1}(1-s)^{\alpha-3} h(s) d s
\end{aligned}
$$

$$
\begin{aligned}
c_{1}= & \frac{1}{6} \frac{1}{\Gamma(\alpha-2)} \int_{0}^{1}(1-s)^{\alpha-3} h(s) d s \\
& -\frac{1}{\Gamma(\alpha)} \int_{0}^{1}(1-s)^{\alpha-1} h(s) d s .
\end{aligned}
$$


Therefore, the unique solution of (5) is

$$
\begin{aligned}
& x(t)= \frac{1}{\Gamma(\alpha)} \int_{0}^{t}(t-s)^{\alpha-1} h(s) d s \\
&+\frac{1}{6} \frac{1}{\Gamma(\alpha-2)} \int_{0}^{1} t(1-s)^{\alpha-3} h(s) d s \\
&-\frac{1}{\Gamma(\alpha)} \int_{0}^{1} t(1-s)^{\alpha-1} h(s) d s \\
&-\frac{1}{6} \frac{1}{\Gamma(\alpha-2)} \int_{0}^{1} t^{3}(1-s)^{\alpha-3} h(s) d s \\
&= \frac{1}{\Gamma(\alpha)} \int_{0}^{t}\left[(t-s)^{\alpha-1}-t(1-s)^{\alpha-1}+\frac{1}{6}(\alpha-2)\right. \\
&+\frac{1}{\Gamma(\alpha)} \int_{t}^{1}\left[-t(1-s)^{\alpha-1}+\frac{1}{6}(\alpha-2)\right. \\
&\left.\times(\alpha-1)\left(t-t^{3}\right)(1-s)^{\alpha-3}\right] h(s) d s \\
&\left.\times\left(t-t^{3}\right)(1-s)^{\alpha-3}\right] h(s) d s .
\end{aligned}
$$

The proof is completed.

Lemma 6. $x(t) \in E$ is a solution to $B V P(1)$, if and only if $x(t)=F x(t)$, where

$$
F x(t)=\int_{0}^{1} G(t, s) f(s, x(s)) d s .
$$

Our main result, based on Schaefer's fixed point theorem, is stated as follows.

Theorem 7. Let $f:[0,1] \times R \rightarrow R$ be continuous. Assume that

(H) there exist nonnegative functions $a, b \in C[0,1]$ such that

$$
|f(t, x)| \leq a(t)+b(t)|x|, \quad \forall t \in[0,1], x \in R
$$

Then, BVP (1) has at least one solution, provided that

$$
\frac{1}{\Gamma(\alpha+1)}\left[2+\frac{\sqrt{3}}{27} \alpha(\alpha-1)\right]\|b\|_{\infty}<1 .
$$

Proof. We divide the proof into the following two steps.

Step 1. F :C[0,1] $\rightarrow C[0,1]$ is completely continuous.

Let $\Omega \subset C[0,1]$ be an open bounded subset. By the continuity of $f$, we can get that $F$ is continuous and $F(\bar{\Omega})$ is bounded. Moreover, there exists a constant $T>0$ such that $|f(t, x)| \leq T, \forall x \in \bar{\Omega}, t \in[0,1]$. Thus, in view of the ArzeláAscoli theorem, we need only to prove that $F(\bar{\Omega}) \subset C[0,1]$ is equicontinuous.
For $0 \leq t_{1} \leq t_{2} \leq 1, x \in \bar{\Omega}$, we have

$$
\begin{aligned}
& \left|F x\left(t_{2}\right)-F x\left(t_{1}\right)\right| \\
& =\mid \int_{0}^{1} G\left(t_{2}, s\right) f(s, x(s)) d s \\
& -\int_{0}^{1} G\left(t_{1}, s\right) f(s, x(s)) d s \mid \\
& \leq T \int_{0}^{1}\left|G\left(t_{2}, s\right)-G\left(t_{1}, s\right)\right| d s \\
& =T \int_{0}^{t_{1}}\left|G\left(t_{2}, s\right)-G\left(t_{1}, s\right)\right| d s \\
& +T \int_{t_{2}}^{1}\left|G\left(t_{2}, s\right)-G\left(t_{1}, s\right)\right| d s \\
& +T \int_{t_{1}}^{t_{2}}\left|G\left(t_{2}, s\right)-G\left(t_{1}, s\right)\right| d s \\
& =\frac{T}{\Gamma(\alpha)} \int_{0}^{t_{1}} \mid\left(t_{2}-s\right)^{\alpha-1}-\left(t_{1}-s\right)^{\alpha-1} \\
& +t_{1}(1-s)^{\alpha-1}-t_{2}(1-s)^{\alpha-1} \\
& +\frac{1}{6}(\alpha-2)(\alpha-1) \\
& \times\left(t_{2}-t_{2}^{3}-t_{1}+t_{1}^{3}\right)(1-s)^{\alpha-3} \mid d s \\
& +\frac{T}{\Gamma(\alpha)} \int_{1}^{t_{2}} \mid t_{1}(1-s)^{\alpha-1}-t_{2}(1-s)^{\alpha-1}+\frac{1}{6}(\alpha-2) \\
& \times(\alpha-1)\left(t_{2}-t_{2}^{3}-t_{1}+t_{1}^{3}\right)(1-s)^{\alpha-3} \mid d s \\
& +\frac{T}{\Gamma(\alpha)} \int_{t_{1}}^{t_{2}} \mid\left(t_{2}-s\right)^{\alpha-1}+t_{1}(1-s)^{\alpha-1}-t_{2}(1-s)^{\alpha-1} \\
& +\frac{1}{6}(\alpha-2)(\alpha-1) \\
& \times\left(t_{2}-t_{2}^{3}-t_{1}+t_{1}^{3}\right)(1-s)^{\alpha-3} \mid d s \\
& \leq \frac{T}{\Gamma(\alpha)} \int_{0}^{t_{1}} \mid\left(t_{2}-s\right)^{\alpha-1}-\left(t_{1}-s\right)^{\alpha-1}+(1-s)^{\alpha-1}\left(t_{1}-t_{2}\right) \\
& +\frac{1}{6}(\alpha-2)(\alpha-1) \\
& \times\left[\left(t_{2}-t_{1}\right)+\left(t_{1}^{3}-t_{2}^{3}\right)\right](1-s)^{\alpha-3} \mid d s \\
& +\frac{T}{\Gamma(\alpha)} \int_{1}^{t_{2}} \mid(1-s)^{\alpha-1}\left(t_{1}-t_{2}\right)+\frac{1}{6}(\alpha-2)(\alpha-1) \\
& \times\left[\left(t_{2}-t_{1}\right)+\left(t_{1}^{3}-t_{2}^{3}\right)\right](1-s)^{\alpha-3} \mid d s
\end{aligned}
$$




$$
\begin{aligned}
& +\frac{T}{\Gamma(\alpha)}\left[3+\frac{2}{3}(\alpha-2)(\alpha-1)\right] \\
& \times\left(t_{2}-t_{1}\right) \longrightarrow 0, \quad t_{2}-t_{1} \longrightarrow 0,
\end{aligned}
$$

which implies that $F(\bar{\Omega}) \subset C[0,1]$ is equicontinuous.

Step 2. A priori bounds.

Set

$$
\Omega=\{x \in C[0,1] \mid x=\lambda F x, \lambda \in(0,1)\} .
$$

Now it remains to show that the set $\Omega$ is bounded.

For $x \in \Omega$, we get $x(t)=\lambda F x(t)$. Thus, form $(H)$, we obtain that

$$
\begin{aligned}
& |x(t)|=\lambda|F x(t)| \\
& \leq\left|\int_{0}^{1} G(t, s) f(s, x(s)) d s\right| \\
& \leq\left|\int_{0}^{1} G(t, s) d s\right|\left(\|a\|_{\infty}+\|b\|_{\infty}\|x\|_{\infty}\right) \\
& =\frac{1}{\Gamma(\alpha)} \mid \int_{0}^{t}\left[(t-s)^{\alpha-1}-t(1-s)^{\alpha-1}\right. \\
& \left.+\frac{1}{6}(\alpha-2)(\alpha-1)\left(t-t^{3}\right)(1-s)^{\alpha-3}\right] d s \\
& +\int_{t}^{1}\left[-t(1-s)^{\alpha-1}+\frac{1}{6}(\alpha-2)(\alpha-1)\right. \\
& \left.\times\left(t-t^{3}\right)(1-s)^{\alpha-3}\right] d s \\
& \times\left(\|a\|_{\infty}+\|b\|_{\infty}\|x\|_{\infty}\right) \\
& =\frac{1}{\Gamma(\alpha)} \mid \frac{1}{\alpha} t^{\alpha}+\frac{1}{\alpha} t(1-t)^{\alpha}-\frac{1}{\alpha} t \\
& +\frac{1}{6}(\alpha-2)(\alpha-1)\left(t-t^{3}\right) \frac{1}{\alpha-2} \\
& -\frac{1}{6}(\alpha-2)(\alpha-1)\left(t-t^{3}\right) \frac{1}{\alpha-2}(1-t)^{\alpha-2} \\
& -\frac{1}{\alpha} t(1-t)^{\alpha}+\frac{1}{6}(\alpha-2)(\alpha-1) \\
& \times\left(t-t^{3}\right) \frac{1}{\alpha-2}(1-t)^{\alpha-2} \\
& \times\left(\|a\|_{\infty}+\|b\|_{\infty}\|x\|_{\infty}\right) \\
& =\frac{1}{\Gamma(\alpha)}\left|\frac{1}{\alpha} t^{\alpha}-\frac{1}{\alpha} t+\frac{1}{6}(\alpha-2)(\alpha-1)\left(t-t^{3}\right) \frac{1}{\alpha-2}\right| \\
& \times\left(\|a\|_{\infty}+\|b\|_{\infty}\|x\|_{\infty}\right) \\
& =\frac{1}{\Gamma(\alpha+1)}\left|t^{\alpha}-t+\frac{1}{6} \alpha(\alpha-1)\left(t-t^{3}\right)\right|
\end{aligned}
$$

$$
\begin{aligned}
& \times\left(\|a\|_{\infty}+\|b\|_{\infty}\|x\|_{\infty}\right) \\
\leq & \frac{1}{\Gamma(\alpha+1)}\left[\left|t^{\alpha}\right|+|t|+\frac{1}{6} \alpha(\alpha-1)\left|t-t^{3}\right|\right] \\
& \times\left(\|a\|_{\infty}+\|b\|_{\infty}\|x\|_{\infty}\right) \\
\leq & \frac{1}{\Gamma(\alpha+1)}\left[2+\frac{\sqrt{3}}{27} \alpha(\alpha-1)\right] \\
& \times\left(\|a\|_{\infty}+\|b\|_{\infty}\|x\|_{\infty}\right), \quad \forall t \in[0,1] .
\end{aligned}
$$

In view of (15), we can see that there exists a constant $M>0$ such that

$$
\|x\|_{\infty} \leq M
$$

As a consequence of Schaefer's fixed point theorem, we deduce that $F$ has a fixed point which is the solution to BVP (1). The proof is completed. results.

Finally, we give an illustrative example to support our new Example 8. Consider the following BVP:

$$
\begin{gathered}
{ }^{c} D_{0+}^{7 / 2} x(t)=2^{t}+4 t^{2} \sin x(t), \quad t \in[0,1], \\
x(0)=x(1)=x^{\prime \prime}(0)=x^{\prime \prime}(1)=0 .
\end{gathered}
$$

A simple calculation shows that $(\Gamma(\alpha+1)) /(2+$ $(\sqrt{3} / 27) \alpha(\alpha-1)) \approx 4.5413$.

Then one can see that

$$
\frac{1}{\Gamma((7 / 2)+1)}\left[2+\frac{\sqrt{3}}{27} \frac{7}{2}\left(\frac{7}{2}-1\right)\right]\left\|4 t^{2}\right\|_{\infty}<1,
$$

which implies that (15) holds. By Theorem 7, BVP (20) has at least one solution.

\section{Conflict of Interests}

The authors declare that there is no conflict of interests regarding the publication of this paper.

\section{Acknowledgments}

This work is supported by the National Natural Science Foundation of China (G61174036), the Scholarship Award for Excellent Doctoral Student granted by the Ministry of Education, and the Graduate Independent Innovation Foundation of Shandong University (yzc10064).

\section{References}

[1] K. B. Oldham and J. Spanier, The Fractional Calculus, Academic Press, New York, NY, USA, 1974.

[2] T. F. Nonnenmacher and R. Metzler, "On the Riemann-Liouvile fractional calculus and some recent applications," Fractals, vol. 3, pp. 557-566, 1995. 
[3] S. G. Samko, A. A. Kilbas, and O. I. Marichev, Fractional Integrals and Derivatives, Theory and Applications, Gordon and Breach, Yverdon, Switzerland, 1993.

[4] X. Xu, D. Jiang, W. Hu, D. O’Regan, and R. P. Agarwal, "Positive properties of Green's function for threepoint boundary value problems of nonlinear fractional differential equations and its applications," Applicable Analysis, vol. 91, no. 2, pp. 323-343, 2012.

[5] X. Xu and X. Fei, “The positive properties of Green's function for three point boundary value problems of nonlinear fractional differential equations and its applications," Communications in Nonlinear Science and Numerical Simulation, vol. 17, no. 4, pp. 1555-1565, 2012.

[6] C. F. Li, X. N. Luo, and Y. Zhou, "Existence of positive solutions of the boundary value problem for nonlinear fractional differential equations," Computers and Mathematics with Applications, vol. 59, no. 3, pp. 1363-1375, 2010.

[7] Z. Bai, "On positive solutions of a nonlocal fractional boundary value problem," Nonlinear Analysis: Theory, Methods and Applications, vol. 72, no. 2, pp. 916-924, 2010.

[8] B. Ahmad and J. J. Nieto, "Existence results for a coupled system of nonlinear fractional differential equations with threepoint boundary conditions," Computers and Mathematics with Applications, vol. 58, no. 9, pp. 1838-1843, 2009.

[9] B. Ahmad and J. J. Nieto, "Boundary value problems for a class of sequential integrodifferential equations of fractional order," Journal of Function Spaces and Applications, vol. 2013, Article ID 149659, 8 pages, 2013.

[10] H. H. Alsulami, S. K. Ntouyas, and B. Ahmad, "Existence results for a Riemann-Liouville-type fractional multivalued problem with integral boundary conditions," Journal of Function Spaces and Applications, vol. 2013, Article ID 676045, 7 pages, 2013.

[11] D. Bǎleanu, O. G. Mustafa, and R. P. Agarwal, "An existence result for a superlinear fractional differential equation," Applied Mathematics Letters, vol. 23, no. 9, pp. 1129-1132, 2010.

[12] Z. Wei, W. Dong, and J. Che, "Periodic boundary value problems for fractional differential equations involving a RiemannLiouville fractional derivative," Nonlinear Analysis: Theory, Methods and Applications, vol. 73, no. 10, pp. 3232-3238, 2010.

[13] X. Yang, Z. Wei, and W. Dong, "Existence of positive solutions for the boundary value problem of nonlinear fractional differential equations," Communications in Nonlinear Science and Numerical Simulation, vol. 17, no. 1, pp. 85-92, 2012.

[14] Y. Zhao, S. Sun, Z. Han, and M. Zhang, "Positive solutions for boundary value problems of nonlinear fractional differential equations," Applied Mathematics and Computation, vol. 217, no. 16, pp. 6950-6958, 2011.

[15] Y. Zhao, S. Sun, Z. Han, and Q. Li, “The existence of multiple positive solutions for boundary value problems of nonlinear fractional differential equations," Communications in Nonlinear Science and Numerical Simulation, vol. 16, no. 4, pp. 2086-2097, 2011.

[16] H. Li, X. Kong, and C. Yu, "Existence of three non-negative solutions for a three point boundary-value problem of nonlinear fractional differential equations," Electronic Journal of Differential Equations, vol. 2012, article 88, 12 pages, 2012.

[17] A. Shi and S. Zhang, "Upper and lower solutions method and a fractional differential equation boundary value problem," Electronic Journal of Qualitative Theory of Differential Equations, vol. 2009, article 30, 13 pages, 2009.
[18] S. Zhang, "Positive solutions for boundary-value problems of nonlinear fractional differential equations," Electronic Journal of Differential Equations, vol. 2006, article 36, 12 pages, 2006.

[19] L. Beghin, "Pseudoprocesses governed by higher-order fractional differential equations," Electronic Journal of Probability, vol. 13, pp. 467-485, 2008.

[20] X. Zhang, L. Liu, and Y. Wu, "The eigenvalue problem for a singular higher order fractional differential equation involving fractional derivatives," Applied Mathematics and Computation, vol. 218, no. 17, pp. 8526-8536, 2012.

[21] C. S. Goodrich, "Existence of a positive solution to a class of fractional differential equations," Applied Mathematics Letters, vol. 23, no. 9, pp. 1050-1055, 2010.

[22] J. R. Graef and L. Kong, "Positive solutions for a class of higher order boundary value problems with fractional q-derivatives," Applied Mathematics and Computation, vol. 218, no. 19, pp. 9682-9689, 2012.

[23] G. Wang, L. Zhang, and S. K. Ntouyas, "Existence of multiple positive solutions of a nonlinear arbitrary order boundary value problem with advanced arguments," Electronic Journal of Qualitative Theory of Differential Equations, vol. 15, pp. 1-13, 2012.

[24] D. Guo and V. Lakshmikantham, Nonlinear Problems in Abstract Cones, Academic Press, New York, NY, USA, 1988. 


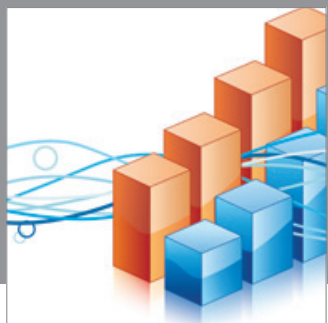

Advances in

Operations Research

mansans

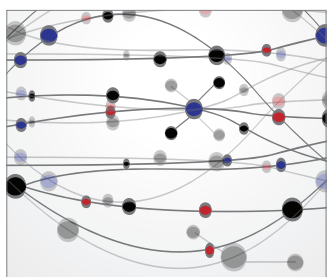

The Scientific World Journal
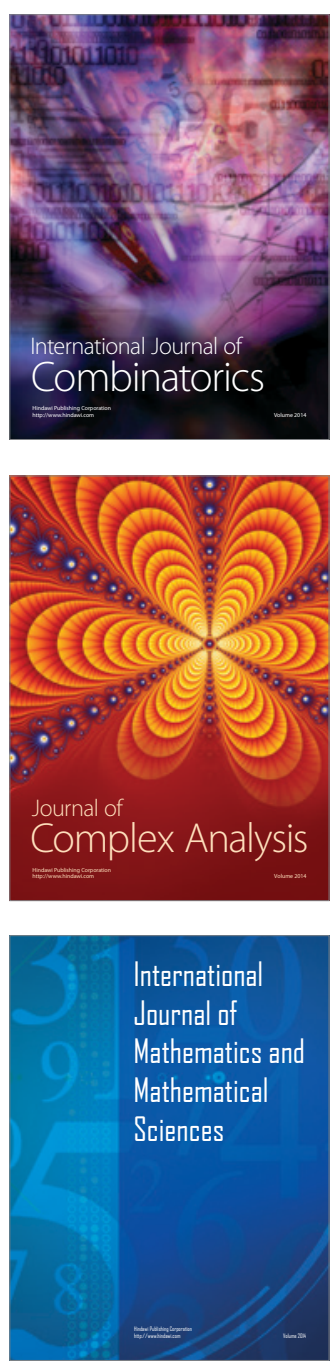
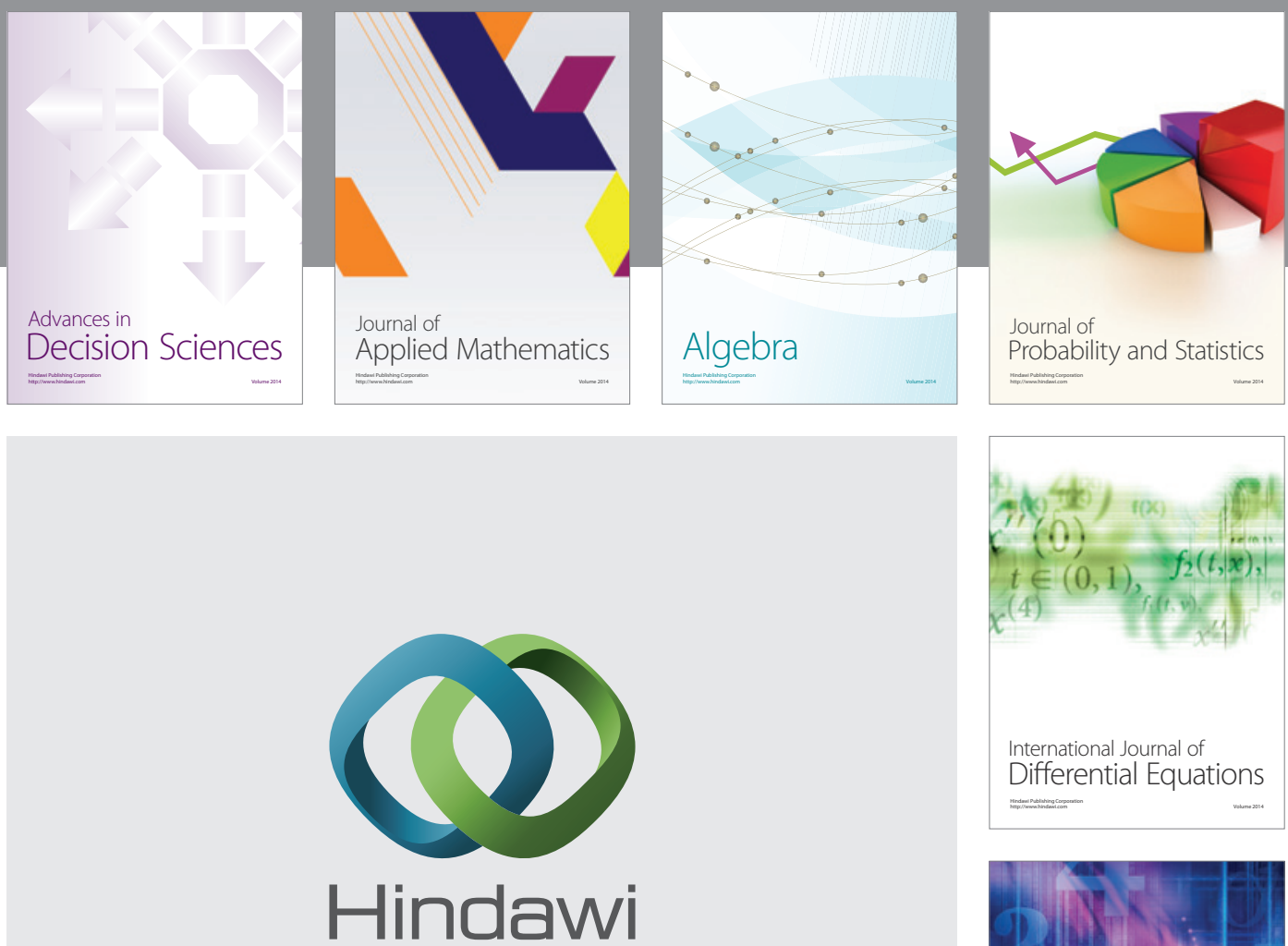

Submit your manuscripts at http://www.hindawi.com
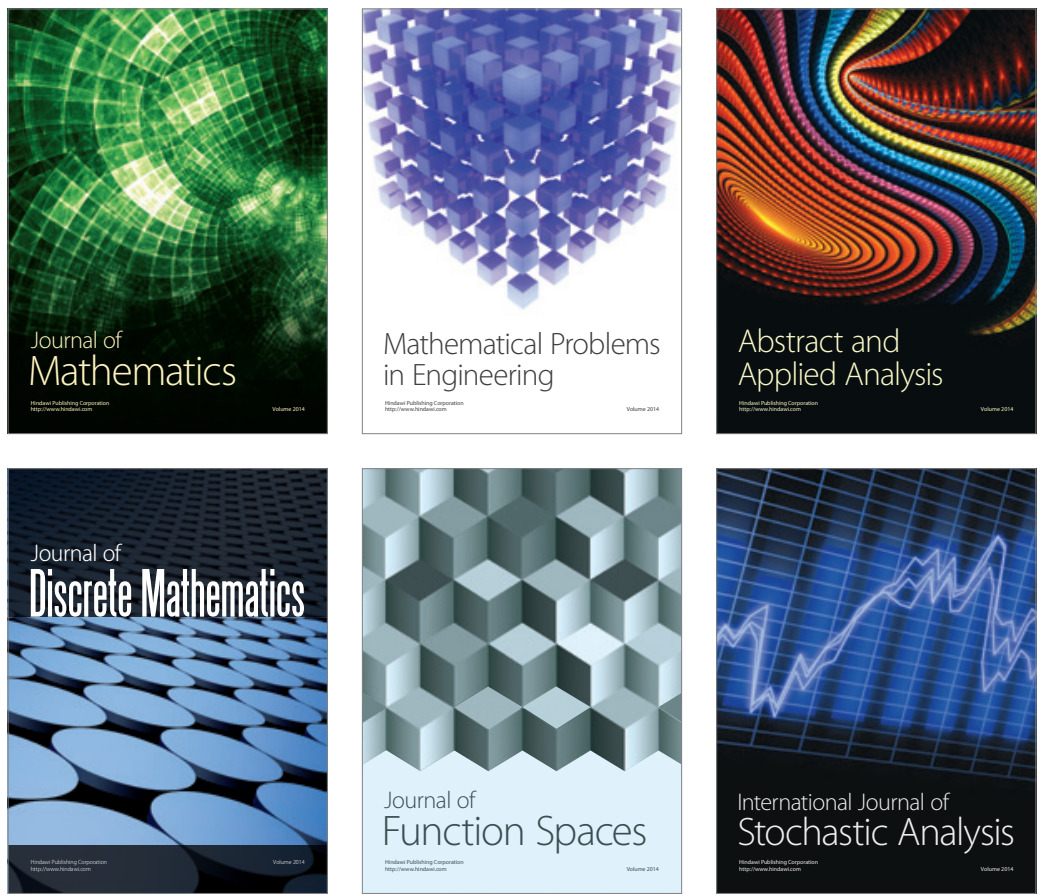

Journal of

Function Spaces

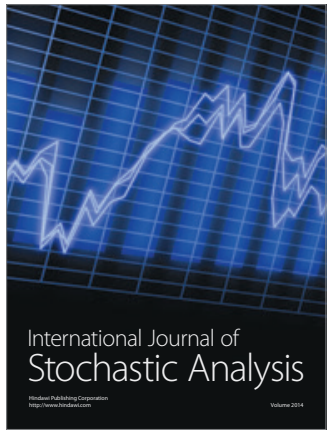

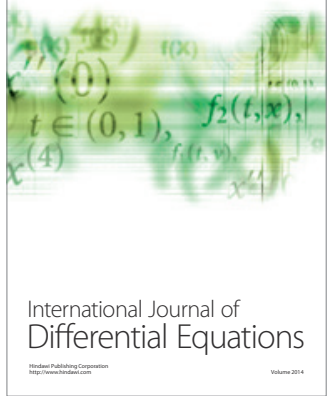
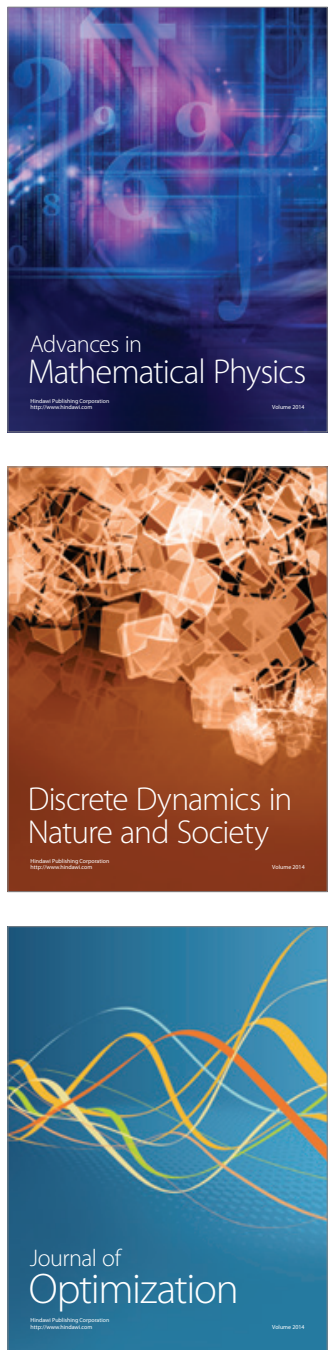